\title{
MANIFESTAÇÕES CLINICAS DA EPILEPSIA TEMPORAL OBSERVADAS DURANTE A ATIVAÇÃO PELO CARDIAZOL
}

\author{
Rubens Moura Ribeiro *
}

As manifestações clínicas de uma crise convulsiva têm, de acôrdo com os conceitos atuais sôbre epilepsia, importância primordial para a localização do foco. Os dados obtidos mediante interrogatório cuidadoso do paciente e seus familiares fornecem elementos para a sistematização de cada tipo de crise convulsiva. Entretanto, êste fato, verdadeiro pelo menos teòricamente, coloca-se muito atrás da realidade prática, já que muitos dos sinais que teriam valor localizatório ocorrem nos primeiros segundos da crise e, em geral, passam despercebidos. Por outro lado, grande número de "auras" não fornece dado localizatório bastante preciso.

A observação, por parte de pessoal experimentado, da crise convulsiva induzida tem a vantagem de fornecer elementos mais precisos e concordes para o diagnóstico localizatório, assim como à análise e interpretação dos fenômenos observados ${ }^{1}$.

Assim, desde que von Meduna ${ }^{34}$, em 1935, introduziu a eletrochoqueterapia no tratamento de esquizofrênicos e verificou a produção de convulsão, a literatura referente ao assunto cada vez mais se enriquece com a colaboração dos que estudam os minimos detalhes da crise convulsiva provocada, quer em animais quer no homem ${ }^{2}, 10,11,28,32$. Entretanto, na grande maioria dos casos, é pela injeção endovenosa de cardiazol que se reproduz, de maneira idêntica, os detalhes da convulsão que o paciente apresenta espontâneamente $7,12,14,35$.

A partir de 1948, com Cure, Rasmussen e Jasper ${ }^{7}$ que, juntamente com Kaufman, Marshall e Walker ${ }^{16}$, foram os precursores do emprêgo do cardiazol (metrazol) para o estudo detalhado das crises epilépticas, o uso desta droga como método de ativação entrou na rotina eletrencefalográfica, seja pela administração oral, mais simples mas de ação retardada $6,15,29$, seja pela via muscular 7, 16. A grande vantagem do cardiazol é poder ser administrado fracionadamente por via venosa ${ }^{24}$, evidenciando a ocorrência de descargas patológicas apenas nos pacientes epilépticos e nunca nos indivíduos normais ${ }^{18}$.

\section{MATERIAL E METODO}

Utilizamos pacientes que, de acôrdo com as informações obtidas, eram portadores de foco temporal. Estes pacientes apresentavam, em geral, crises de dificil contrôle, com manifestaçóes clínicas que se repetiam várias vêzes por mess ou mesmo

Trabalho da Clínica Neurológica da Fac. Med. de Ribeirão Prêto, da Univ. de Sāo Paulo (Prof. Jorge Armbrust-Figueiredo): * Assistente. 
por semana. Os pacientes eram, então, encaminhados à prova do cardiazol, com suspeita clinica bem definida quanto ao diagnóstico topográfico. Não nos preocupamos em analisar as prováveis causas etiológicas, sendo porém excluidos os pacientes com tumores cerebrais ou com distúrbios cérebro-vasculares.

Procedemos à ativação pelo cardiazol com a finalidade de reproduzir os sintomas e sinais e, posslvelmente, a própria crise clinica. As diferentes manifestaçōes foram registradas de acôrdo com a sequienncia de sua eclosão. Nosso intuito fol estudar a seqüéncia do aparecimento dos sinais à medida que a descarga elétrica anormal e excessiva se propagava às áreas vizlnhas, verificando de maneira detalhada a "marcha" da epilepsia temporal. Nosso plano foi estabelecer as diferentes sequiências da crise, de acôrdo com a situação topográfica do foco epiléptico no lobo temporal.

Nosso material consta de 25 pacientes que foram submetidos à ativação pelo cardiazol, por administração endovenosa de $50 \mathrm{mg}$ cada 30 segundos, até um total de $500 \mathrm{mg}$, de acôrđo com a técnica usada por váríos autores ${ }^{5,9,18,10,26,27}$.

Durante a injeção, o paciente permanecia em decúbito dorsal e com os olhos fechados. O eletrencefalograma era registrado ininterruptamente durante todo o tempo de aplicação do cardiazol, com o papel de registro correndo na velocidade de $3 \mathrm{~cm}$ por segundo em aparelho Grass de 8 canals. A colocação dos eletrodos obedeceu à técnica do Montreal Neurological Institute. Durante a administração do cardiazol o paciente era submetido a contínuo interrogatório, com a finalidade de se detectar qualquer manifestação inicial de crise ou distúrbio de consciência.

o registro das eventuais manifestações decorrentes da ativação foi feito diretamente sóbre o traçado eletrencefalográfico, com o intuito de correlacionar mais precisamente os sinais clínicos com a dose de cardiazol injetada e o tempo de duração do fenômeno, bem como a sua seqüência.

Esses pacientes apresentaram, ao exame eletrencefalográfico, focos convulsiógenos ativos cujas localizações obedeceram à orientação seguida por Paulo Pinto Pupo ${ }^{27}$, que considera: 1) foco superficial, caracterizado por descargas paroxísticas de espículas ou onda "sharp" de projeção em áreas restritas do córtex cerebral, sem repercussão para o hemisfério oposto; 2) foco profundo, caracterizado por descargas de projeção em áreas não restritas, difundindo-se mais ou menos às áreas vizinhas e, também, às áreas homólogas do hemisfério oposto.

Para a análise do material organizamos quadros nos quais as diferentes manifestações clínicas são registradas por grupos de sintomas de acôrdo com ficha especialmente elaborada para êsse fím ${ }^{3}$.

\section{RESULTADOS}

Os achados registrados na seqüência eletrencefalográfica correspondem a cada uma das manifestações clínicas relatadas pelo paciente ou observada pelo médico, conforme a referência numérica correspondente (quadros 1, 2, 3,4 e 5 ).

A análise do material sugere que existe correlação na seqüência das manifestações clínicas obtidas através da ativação pelo cardiazol e o aparecimento das alterações registradas pelo EEG concomitantemente.

No quadro 1 , em que reunimos 13 pacientes portadores de manifestações de tipo vegetativo, verificamos que $11(\operatorname{casos} 1,2,7,9,10,12,13,18$, 21, 24 e 25), ou seja, 84,6\%, apresentavam alterações eletrencefalográficas que se iniciavam por descargas de projeção nas porções anteriores de um dos lobos temporais, e, em dois (casos 15 e 16), ou seja, 15,4\%, essas manifestações se iniciaram por descargas de projeção nas porções médias de 


\begin{tabular}{|c|c|c|}
\hline Casos & Manifestacões clinicas & Seqüência do EEG \\
\hline 1 & $\begin{array}{l}\text { 1. Sensação cardiaca } \ldots \ldots \ldots \ldots \ldots \ldots \ldots \ldots \\
\text { 2. Distúrbios de consciência } \ldots \ldots \ldots \ldots \ldots \ldots \\
\text { 3. Automatismo complexo } \ldots \ldots \ldots \ldots \ldots \ldots\end{array}$ & $\begin{array}{l}\text { 1. F7 } \\
\text { 2. F7, T3 } \\
\text { 3. F3, F7, T3, T5 }\end{array}$ \\
\hline 2 & $\begin{array}{l}\text { 1. Sensação de cefaléia } \ldots \ldots \ldots \ldots \ldots \ldots \ldots \\
\text { 2. Distúrbio de consciéncia } \ldots \ldots \ldots \ldots \ldots \ldots \\
\text { 3. Palidez } \ldots \ldots \ldots \ldots \ldots \ldots \ldots \ldots \ldots \ldots \ldots \ldots\end{array}$ & $\begin{array}{lll}\text { 1. } & \text { F7 } & \\
\text { 2. F7, T3 } \\
\text { 3. F7, T3, T5 }\end{array}$ \\
\hline 7 & $\begin{array}{l}\text { 1. Mal-estar geral } \ldots \ldots \ldots \ldots \ldots \ldots \ldots \ldots \\
\text { 2. Distúrbio de consciéncia } \ldots \ldots \ldots \ldots \ldots \ldots \\
\text { 3. Movimentos de sucção } \ldots \ldots \ldots \ldots \ldots \ldots\end{array}$ & $\begin{array}{l}\text { 1. F7 } \\
\text { 2. F7, T3, T5 } \\
\text { 3. F7, T3, T5 - difusa }\end{array}$ \\
\hline 9 & $\begin{array}{l}\text { 1. Mal-estar geral } \ldots \ldots \ldots \ldots \ldots \ldots \ldots \ldots \\
\text { 2. Distúrbios de consciência } \ldots \ldots \ldots \ldots \ldots \ldots \\
\text { 3. Automatismo complexo } \ldots \ldots \ldots \ldots \ldots \ldots\end{array}$ & $\begin{array}{ll}\text { 1. } & \text { F3, F7 } \\
\text { 2. F3, F7, T3 } \\
\text { 3. F3, F7, T3 - difusa }\end{array}$ \\
\hline 10 & $\begin{array}{l}\text { 1. Sensação gástrica } \ldots \ldots \ldots \ldots \ldots \ldots \ldots \ldots \\
\text { 2. Distúrbios de consciência } \ldots \ldots \ldots \ldots \ldots \ldots \\
\text { 3. Crise de riso } \ldots \ldots \ldots \ldots \ldots \ldots \ldots \ldots \ldots \ldots \ldots\end{array}$ & $\begin{array}{l}\text { 1. F7 } \\
\text { 2. F7, T3 } \\
\text { 3. F3, F7, T3, T5 }\end{array}$ \\
\hline 12 & $\begin{array}{l}\text { 1. Mal-estar geral } \ldots \ldots \ldots \ldots \ldots \ldots \ldots \ldots \\
\text { 2. Distúrbios de consciência } \ldots \ldots \ldots \ldots \ldots \ldots \\
\text { 3. } \quad \text { Automatismo } \ldots \ldots \ldots \ldots \ldots \ldots \ldots \ldots \ldots\end{array}$ & $\begin{array}{lll}\text { 1. } & \text { F7, } & \text { F3 } \\
\text { 2. F7, } & \text { T3 - - difusa } \\
\text { 3. F7, } & \text { T3 }\end{array}$ \\
\hline 13 & $\begin{array}{l}\text { 1. } \quad \text { Mal-estar geral } \ldots \ldots \ldots \ldots \ldots \ldots \ldots \ldots \\
\text { 2. Distúrbios de consciência } \ldots \ldots \ldots \ldots \ldots \ldots \\
\text { 3. Deglutição } \ldots \ldots \ldots \ldots \ldots \ldots \ldots \ldots \ldots \ldots\end{array}$ & $\begin{array}{lll}\text { 1. } & \text { T3, T5 } \\
\text { 2. } & \text { F7, T3, T5 } \\
\text { 3. } & \text { F7, T3, T5 }\end{array}$ \\
\hline 15 & $\begin{array}{l}\text { 1. Mal-estar geral } \ldots \ldots \ldots \ldots \ldots \ldots \ldots \ldots \\
\text { 2. Sem distúrbio de consciência } \ldots \ldots \ldots \ldots\end{array}$ & $\begin{array}{l}\text { 1. } \mathrm{T} 4 \\
\text { 2. } \mathrm{F} 4, \mathrm{~F} 8, \mathrm{~T} 4\end{array}$ \\
\hline 16 & $\begin{array}{l}\text { 1. Sensação cardiaca } \ldots \ldots \ldots \ldots \ldots \ldots \ldots \\
\text { 2. Sem distúrbio de consciência } \ldots \ldots \ldots \ldots\end{array}$ & $\begin{array}{l}\text { 1. } \mathrm{F} 8 \\
\text { 2. } F 8, T 4-\text { difusa }\end{array}$ \\
\hline 18 & $\begin{array}{l}\text { 1. Mal-estar geral } \ldots \ldots \ldots \ldots \ldots \ldots \ldots \ldots \\
\text { 2. Distúrbios de consciência } \ldots \ldots \ldots \ldots \ldots \ldots \\
\text { 3. Automatismo complexo } \ldots \ldots \ldots \ldots \ldots \ldots\end{array}$ & $\begin{array}{l}\text { 1. F7 } \\
\text { 2. F7, T3 } \\
\text { 3. F7, T3 -- difusa }\end{array}$ \\
\hline 21 & $\begin{array}{l}\text { 1. Mal-estar geral } \ldots \ldots \ldots \ldots \ldots \ldots \ldots \ldots \\
\text { 2. Distúrbios de consciência } \ldots \ldots \ldots \ldots \ldots\end{array}$ & $\begin{array}{l}\text { 1. } \mathrm{F7} \\
\text { 2. F7, T3, T5, F8 }\end{array}$ \\
\hline 24 & $\begin{array}{l}\text { 1. Cianose } \ldots \ldots \ldots \ldots \ldots \\
\text { 2. Distúrbios de consciência } \ldots \ldots \ldots \ldots \ldots \ldots \\
\text { 3. Crise motora focal } \ldots \ldots \ldots \ldots \ldots \ldots \ldots\end{array}$ & $\begin{array}{l}\text { 1. F7 } \\
\text { 2. F7, T3 } \\
\text { 3. F3, F7, T5 }\end{array}$ \\
\hline 25 & $\begin{array}{l}\text { 1. Mal-estar geral } \ldots \ldots \ldots \ldots \ldots \ldots \ldots \ldots \\
\text { 2. Distúrbios de consciência } \ldots \ldots \ldots \ldots \ldots \ldots \\
\text { 3. Crise mastigatória } \ldots \ldots \ldots \ldots \ldots \ldots \ldots\end{array}$ & $\begin{array}{ll}\text { 1. } & \mathrm{F} 8 \\
\text { 2. } & \mathrm{F} 4, \mathrm{~F} 8, \mathrm{~T} 4, \mathrm{~T} 6 \\
\text { 3. } & \mathrm{F} 4, \mathrm{~F} 8, \mathrm{~T} 4, \mathrm{~T} 6\end{array}$ \\
\hline
\end{tabular}




\begin{tabular}{|c|c|c|}
\hline Caso & Manifestacões clinicas & Seqüuência do EEC \\
\hline 6 & $\begin{array}{l}\text { 1. Déjà vu } \ldots \ldots \ldots \ldots \ldots \ldots \ldots \ldots \ldots \ldots \\
\text { 2. Sem distúrbio de consciência } \ldots \ldots \ldots \ldots \ldots \ldots \ldots \ldots \ldots \ldots\end{array}$ & $\begin{array}{l}\text { 1. } \mathrm{F} 8 \\
\text { 2. } \mathrm{F} 8, \mathrm{~T} 4 \\
\text { 3. }\end{array}$ \\
\hline
\end{tabular}

Quadro 2 - Seqüência das manifestaçōes clinicas e eletrencefalográficas em um paciente com crises psíquicas.

\begin{tabular}{|c|c|c|}
\hline Caso & Manifestações clinicas & Seqülência do EEG \\
\hline 17 & $\begin{array}{l}\text { 1. Crise motora facial direita } \ldots \ldots \ldots \ldots \\
\text { 2. Distúrbio de consciência } \ldots \ldots \ldots \ldots \ldots \\
\text { 3. Crise automática complexa } \ldots \ldots \ldots \ldots\end{array}$ & $\begin{array}{l}\text { 1. F7 } \\
\text { 2. F7, T3, T5 } \\
\text { 3. F3, F7, T3, T5 }\end{array}$ \\
\hline
\end{tabular}

Quàlro 3 - Seqüência das manifestações clinicas e eletrencefalográficas em um paciente com crises motoras.

\begin{tabular}{|c|c|c|}
\hline Caso & Manifestaçōes clinicas & Seqü̈encia do EEG \\
\hline 22 & $\begin{array}{l}\text { 1. Ausência tipo } \mathbf{P M} \ldots \ldots \ldots \ldots \ldots \ldots \ldots \ldots \ldots \ldots \ldots \ldots \ldots \ldots \\
\text { 2. Crise motora } \ldots \ldots \ldots \ldots \ldots \ldots \ldots \ldots\end{array}$ & $\begin{array}{l}\text { 1. T3 } \\
\text { 2. T3, F7, T5 }\end{array}$ \\
\hline 4 & $\begin{array}{l}\text { 1. Ausência tipo } P M \ldots \ldots \ldots \ldots \ldots \ldots \ldots \\
\text { 2. Automatismo complexo } \ldots \ldots \ldots \ldots \ldots \ldots \ldots\end{array}$ & $\begin{array}{l}\text { 1. } \mathrm{T} 4 \\
\text { 2. } \mathrm{T} 4, \mathrm{~F} 8, \mathrm{~T} 6\end{array}$ \\
\hline
\end{tabular}

Quadro 4 - Seqüência das manifestações clínicas e eletrencefalográficas em dois purientes com crises de "ausência". 


\begin{tabular}{|c|c|c|}
\hline Caso & Manifestaçöes clinicas & Sequïencia do $E E G$ \\
\hline 3 & $\begin{array}{l}\text { 1. Manifestação visual } \ldots \ldots \ldots \ldots \ldots \ldots \ldots \\
\text { 2. Distúrbio de consciência } \ldots \ldots \ldots \ldots \ldots \ldots \\
\text { 3. Crise motora focal } \ldots \ldots \ldots \ldots \ldots \ldots \ldots\end{array}$ & $\begin{array}{ll}\text { 1. } & \text { T5, T3 } \\
\text { 2. } & \text { T5, T3 } \\
\text { 3. } & \text { F3, F7, T3, T5 }\end{array}$ \\
\hline 5 & $\begin{array}{l}\text { 1. Manifestacão visual } \ldots \ldots \ldots \ldots \ldots \ldots \ldots \\
\text { 2. Distúrbio de consciência } \ldots \ldots \ldots \ldots \ldots \ldots \\
\text { 3. Crise mastigatória } \ldots \ldots \ldots \ldots \ldots \ldots\end{array}$ & $\begin{array}{ll}\text { 1. } & \text { F7 } \\
\text { 2. F7, T3, Г5 } \\
\text { 3. F3, F7, T3, T5 }\end{array}$ \\
\hline 8 & $\begin{array}{l}\text { 1. Sensaçāo parestésica } \ldots \ldots \ldots \ldots \ldots \ldots \ldots \\
\text { 2. Sem distúrbio de consciência } \ldots \ldots \ldots \ldots \\
\text { 3. Angústia } \ldots \ldots \ldots \ldots \ldots \ldots \ldots \ldots \ldots \ldots \ldots \ldots\end{array}$ & $\begin{array}{ll}\text { 1. } & \text { T3 } \\
\text { 2. } & \text { T3, T5, F7 } \\
\text { 3. } & \text { F7, T3, T5 }\end{array}$ \\
\hline 11 & $\begin{array}{l}\text { 1. Sensação parestésica } \ldots \ldots \ldots \ldots \ldots \ldots \ldots \\
\text { 2. Distúrbio de consciência } \ldots \ldots \ldots \ldots \ldots \ldots \\
\text { 3. Crìse de sucção } \ldots \ldots \ldots \ldots \ldots \ldots \ldots \ldots \ldots \ldots\end{array}$ & $\begin{array}{ll}\text { 1. } & \text { F7 } \\
\text { 2. } & \text { F7, T3, T5 } \\
\text { 3. F7, T3, T5 }\end{array}$ \\
\hline 19 & $\begin{array}{l}\text { 1. Sensacăo gustativà } \ldots \ldots \ldots \ldots \ldots \ldots \ldots \\
\text { 2. Sem distúrbio de consciência } \ldots \ldots \ldots \ldots\end{array}$ & $\begin{array}{l}\text { 1. } \mathrm{T} 4 \\
\text { 2. } \mathrm{T} 4, \mathrm{~F} 8\end{array}$ \\
\hline 20 & $\begin{array}{l}\text { 1. Sensação parestésica } \ldots \ldots \ldots \ldots \ldots \ldots \\
\text { 2. Sem distúrbio de consciência } \ldots \ldots \ldots \ldots\end{array}$ & $\begin{array}{llll}\text { 1. } & \text { F7 e } & \text { F8 } \\
\text { 2. } & \text { F7 } & \text { e } & \text { F8 }\end{array}$ \\
\hline 23 & $\begin{array}{l}\text { 1. Sensação vertiginusa } \ldots \ldots \ldots \ldots \ldots \ldots \ldots \\
\text { 2. Distúrbio de consciência } \ldots \ldots \ldots \ldots \ldots \ldots \\
\text { 3. Crise mastigatória } \ldots \ldots \ldots \ldots \ldots \ldots\end{array}$ & $\begin{array}{l}\text { 1. F7 e F8 } \\
\text { 2. F7 } \\
\text { 3. F7 - difusa }\end{array}$ \\
\hline
\end{tabular}

Quadio 5 - Seqüência das manifestacões clinicas e eletrencefalogrificas em sete pacientes com irises sensoriais.

\begin{tabular}{c|c|c}
\hline Porcito anterior do lobo temporal & Porcūo média lobo temporal \\
Foco superficial (7 casus) & Foco profundo $(4$ casos $)$ & Foco superficial (2 (asos)
\end{tabular}

Quadro 6 - Situaçio do foco quanto à topografia e profundidade em 13 pacientes com manifestações vegetativas. 
um dos lobos temporais (quadro 6). Em 9 pacientes (casos 1, 2, 10, 15, 16, $18,21,24$ e 25) o foco era superficial; em 4 (casos $7,9,12$ e 15) o foco era profundo (quadro 6). Dos 13 casos dêste grupo, 10 (76,9\%) apresentavam descargas no lobo temporal esquerdo e $3(23,1 \%)$ no lobo temporal direito. Após as primeiras descargas eletrencefalográficas o traçado apresentou alterações difusas em 9 (casos $1,7,9,12,13,16,18,24$ e 25), ou seja $69,2 \%$ e permaneceu com alterações focais em 4 , ou seja $30,8 \%$ (casos $2,10,15$ e 21).

Em 11 pacientes $(84,6 \%)$ foi verificado distúrbio de consciência logo após a manifestação inicial; em $2(15,4 \%)$ não houve distúrbio de consciência, apesar de se ter atingido a dose máxima proposta de cardiazol (500 mg).

Se considerarmos a incidência das manifestações iniciais nos pacientes portadores de crises convulsivas vegetativas verificamos que o sintoma subjetivo que predominou foi a queixa de mal-estar geral ou indefinido, que apareceu em 8 pacientes $(61,5 \%)(\operatorname{casos} 7,9,12,13,15,18,21$ e 25). Dêstes 8 casos, 4 apresentavam foco superficial (casos $7,9,12$ e 13) e 4 apresentavam foco profundo (casos 15, 18, 21 e 25).

As manifestações cardíacas, caracterizadas por taquicardia, foram relatadas por dois pacientes $(15,4 \%)$ como manifestação inicial (casos 1 e 16). Esses dois pacientes apresentaram, no EEG, disritmia por onda "sharp" de projeção cortical em áreas do lobo temporal, sendo que em um caso no lobo temporal esquerdo e, no outro, no lobo temporal direito; um dêles (caso 1) apresentou distúrbio de consciência e o outro não (caso 16).

Finalmente, como manifestações menos freqüentes, encontramos relatada uma única vez manifestação cefálica definida como cefaléia difusa, de caráter paroxístico, manifestação gástrica definida como "coisa ruim no estômago" e alterações vasomotoras caracterizadas por cianose intensa. Os 3 pacientes apresentaram, após a manifestação inicial, distúrbio de consciência e o EEG evidenciou foco cortical de projeção em áreas anteriores do lobo temporal esquerdo.

Da análise geral do quadro 1 verificamos que, após a manifestação inicial e o distúrbio de consciência, a manifestação clínica predominante foi o automatismo psicomotor em suas variadas formas (sucção, mastigação, deglutição, etc.). Não nos baseamos nas manifestações psicomotoras para a classificação dêste grupo já que as crises dêsse tipo podem depender também de focos situados nos lobos frontal e parietal, na região centrencefálica ${ }^{22}$ ou em outras regiōes ligadas funcionalmente ao lobo temporal ${ }^{8}$, apesar de Gibbs e col. ${ }^{13}$ encontrarem maior incidência de crises de tipo psicomotor em pacientes com foco na parte anterior do lobo temporal. A maioria dos nossos pacientes que apresentavam manifestações psicomotoras após a manifestação inicial evidenciou foco de projeção nesta topografia.

No quadro 2 analisamos as manifestaçōes psíquicas. Um dos pacientes (caso 6) apresentou sensação de estranheza (jamais vu) durante a administração do cardiazol, sem, entretanto, apresentar distúrbio complexo de consciência. A seqüência eletrencefalográfica revelou um foco convulsióge- 
no cortical de projeção em área anterior do lobo temporal direito, com propagação para área mais posterior.

Estudando 1.000 casos de epilepsia temporal, Armbrust-Figueiredo ${ }^{3}$ referiu a sensação de estranheza como manifestação epiléptica do lobo temporal em $9 \%$ de seus pacientes, a maioria dêles com foco situado em áreas anteriores do lobo temporal.

Não há mais dúvida da importância que o lobo temporal ou as estruturas a êle ligadas através do fórnix e formação hipocampal representam no mecanismo da memória e, portanto, na evocação de experiências anteriormente vividas ${ }^{33}$, simulando não raramente síndromes psíquicas complexas. Mulder e Daly ${ }^{20}$, em 100 pacientes com epilepsia temporal, verificaram que as queixas mais usuais eram do âmbito psiquiátrico; Weil ${ }^{36}$ admite a existência de lesões com localização precisa no lobo temporal em pacientes com queixas de mêdo, de experiências irreais, etc.

As crises motoras faciais (quadro 3) decorrentes de lesão no lobo temporal não mereceram durante muito tempo a atenção dos autores. Somente em 1944 com Baldwin e col. ${ }^{4}$ é que foram descritas as primeiras crises com movimentos faciais, decorrentes de estimulação de estruturas ligadas ao lobo temporal, principalmente o núcleo amigdalóide. Tivemos a oportunidade de observar um paciente (caso 17) portador de crise motora facial direita que, na seqüência eletrencefalográfica, apresentava foco cortical de localização inicial em áreas anteriores do lobo temporal esquerdo, com propagação ulterior para as áreas posteriores.

Crises de ausência ocorreram como manifestação objetiva inicial em dois pacientes (casos 4 e 22). No estudo eletrencefalográfico foi verificado foco convulsiógeno cortical em áreas médias do lobo temporal com propagação para as regiões anteriores e posteriores. Em um dos pacientes havia comprometimento do lobo temporal direito (caso 4) e, no outro, do lobo temporal esquerdo (caso 22). Foi a partir da verificação de lesão temporal em pacientes portadores de "epilepsia idiopática" que os autores começaram a dispensar maior atenção ao estudo da epilepsia centrencefálica por foco temporal ${ }^{31}$.

Manifestações sensoriais (quadro 5) foram referidas por 7 de nossos pacientes (casos 3, 5, 8, 11, 19, 20 e 23) como manifestação inicial após a administração do cardiazol, e incluíram manifestações visuais, parestésicas, gustativas e vestibulares. As sensações parestésicas foram verificadas em 3 pacientes (casos 8, 11 e 20) e, em um dêles, precederam crise do tipo psicomotor (caso 11); nos 3 pacientes o foco convulsiógeno era cortical e em dois estava localizado em áreas anteriores do lobo temporal esquerdo, com propagação para as áreas posteriores em um dêles (caso 11) e com repercussão bilateral em outro (caso 20); no terceiro paciente (caso 8) o foco estava localizado em áreas médias do lobo temporal esquerdo e se propagava para as áreas posteriores e anteriores.

Manifestações visuais foram referidas por dois pacientes (casos 3 e 5) como fenômeno inicial de crise após a administração do cardiazol: um (caso 3) referiu ver bolas coloridas e o outro (caso 5) teve a impressão 


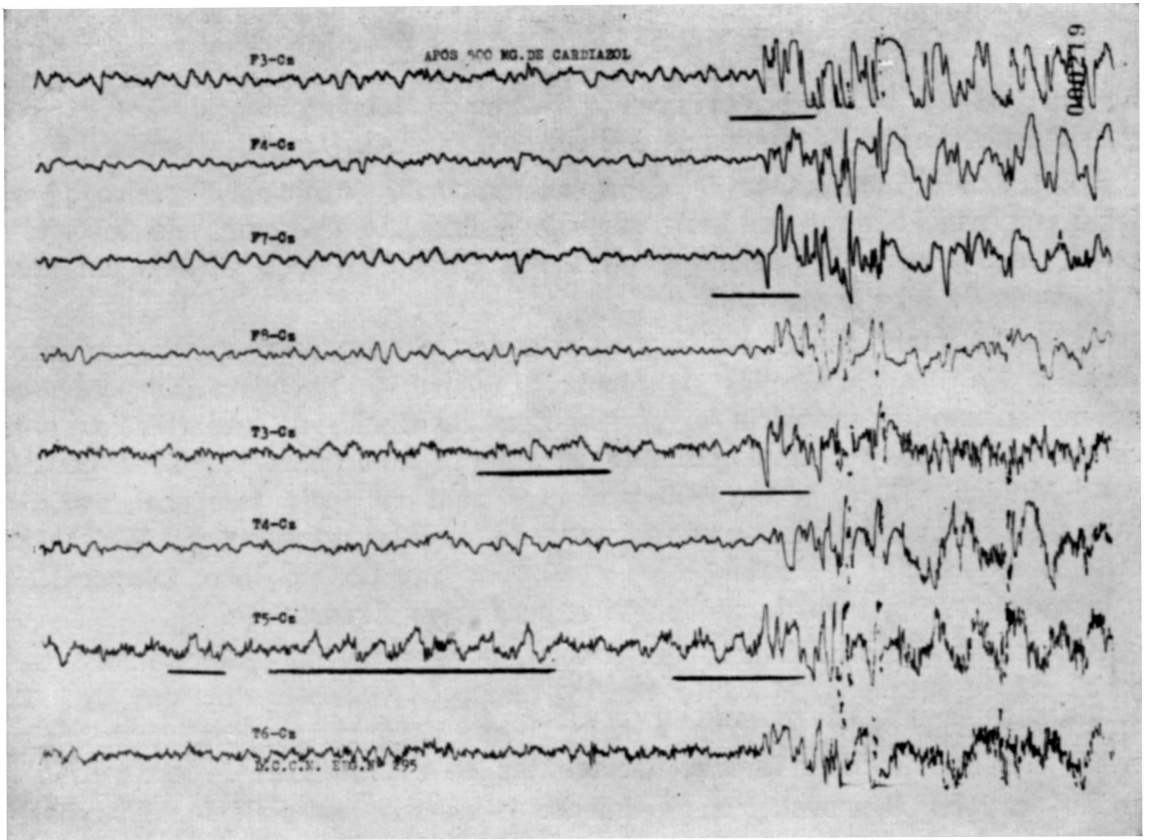

Fig. 1 - Caso s (EEG no 295). Onda "sharp" ritmada de projeçõo inicial em áreas posteriores do lobo temporal esquerdo; em seguida há propagaç̄o da descarga para áreas médias $e$ imediata alteraçõo difusa do traçado.

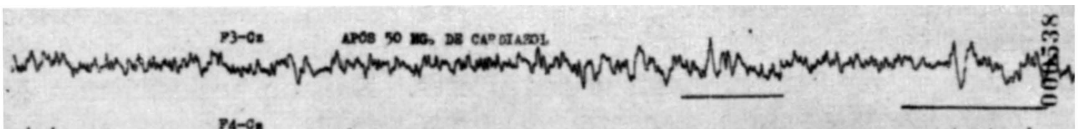

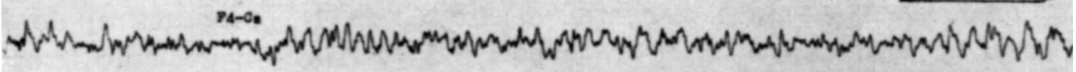

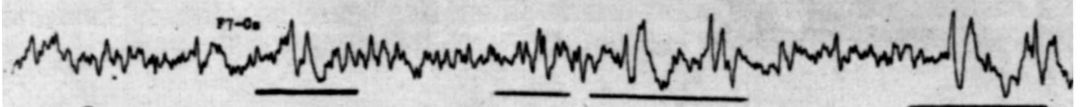

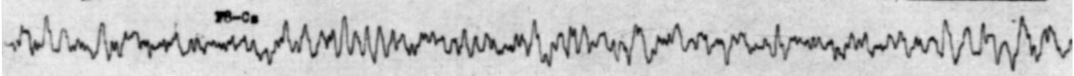

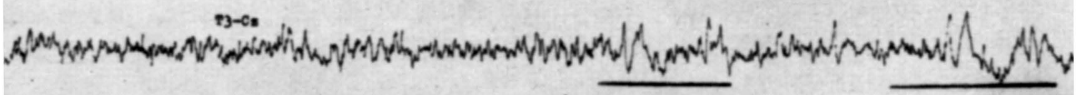

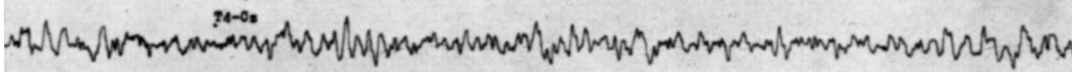

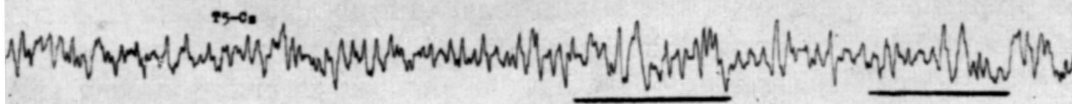

Fig. 2 - Caso 5 (EEG n" 308). Onda "sharp" de projeçāo inicial em âreas anteriores do lobo temporal esquerdo; em seguida há propagacõo da descarga para áreas médias e posteriores. 


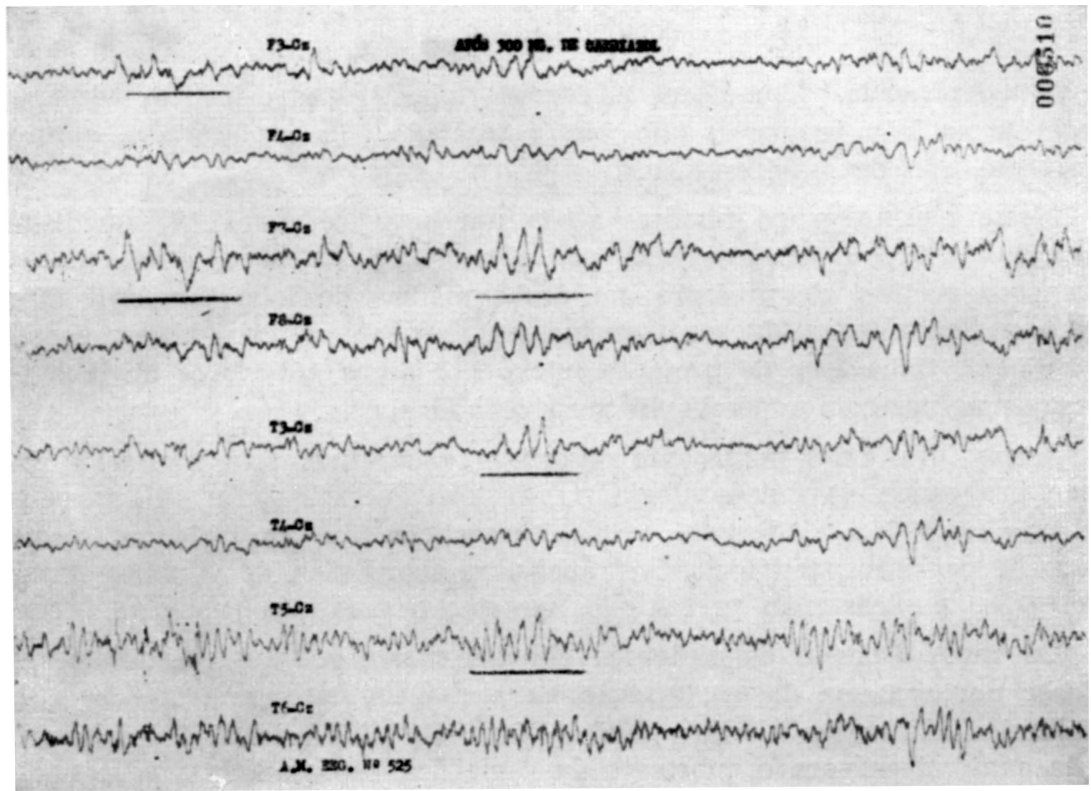

Fig. 3 - Caso 11 (EEG n" 525). Onda "sharp" ritmada de projecão inicial em áreas anteriores do lobo temporal esquerdo; em seguida há propagacão para áreas médias e posteriores.

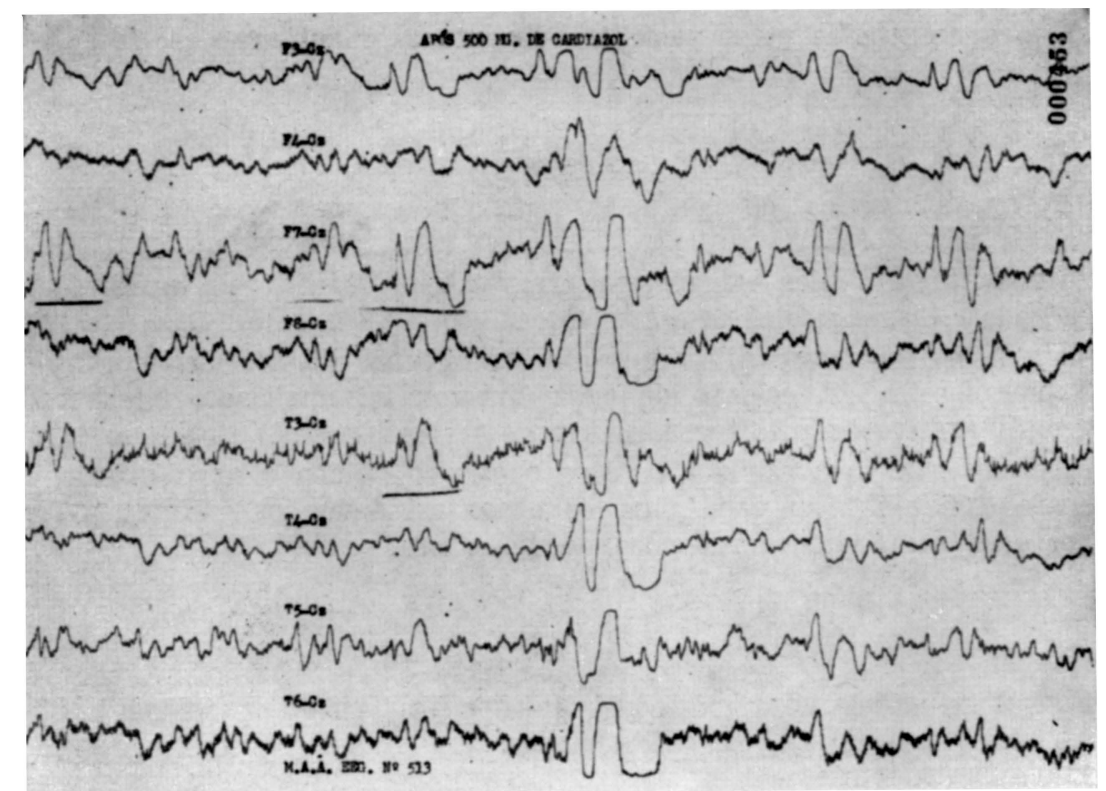

Fig. 4-Caso 21 (EEG no 513). Onda "sharp" de projecăo inicial em áreas anteriores do lobo temporal esquerdo; em seguida há propagação da descarga para áreas médias e imediata alteração difusa do traçado. 
de vista embaçada. Um dêstes pacientes (caso 3) tinha foco de localização posterior no lobo temporal, fato êsse assinalado pela maioria dos autores ${ }^{23}$ para êste tipo de manifestação.

Neste mesmo grupo revimos ainda um paciente (caso 19) que referiu sensação gustativa, caracterizada por gôsto ruim na bôca, e que apresentava foco cortical de projeção em áreas médias do lobo temporal direito. Outro paciente (caso 23), com queixa vestibular caracterizada por sensação vertiginosa, tinha foco de projeção inicial em áreas anteriores do lobo temporal esquerdo, com repercussão para o lado oposto.

Nosso intuito foi puramente descritivo e objetivo, sem pretender esclarecer problemas de fisiopatologia ou de eletrencefalografia relacionadas à epilepsia temporal. Ademais, nossa observação foi baseada em pequeno grupo de pacientes, sendo possivel apenas a abordagem de algumas das manifestações clínicas, não permitindo apreciação geral do problema.

Da multiplicidade de sintomas clinicos tornou-se possivel, assim, estabelecer um esquema de organização na seqüência das manifestações e concomitantes alterações eletrencefalográficas. Em 1937, Papez ${ }^{21}$ elaborou as bases anatômicas para o substrato das emoções, colocando sua sede no lobo temporal e, em 1957, Stevens ${ }^{30}$ chamou a atenção para a possibilidade de ser mapeado um homúnculo psiquico, de maneira idêntica ao homúnculo descrito na marcha de epilepsia jacksoniana. Estudos ulteriores são necessários para maior elucidação e complementação do assunto.

Os dados colhidos de nosso material sugerem haver uma seqüência ordenada, tanto das manifestações clínicas como das alterações eletrencefalográficas apresentadas pelos pacientes durante as manifestações da epilepsia temporal.

\section{RESUMO}

Foram analisados, do ponto de vista clínico e eletrencefalográfico, 25 pacientes portadores de epilepsia temporal com a finalidade de comparar a sintomatologia clínica evidenciada através da ativação pelo cardiazol com os achados eletrencefalográficos obtidos simultâneamente. Para facilidade do estudo, os pacientes foram divididos em grupos de acôrdo com a sintomatologia clínica. A eclosão de cada sintoma foi analisada objetivamente utilizando a ativação pelo cardiazol em doses fracionadas. Com isso foi possível estabelecer uma seqüência entre o quadro clínico e as alterações eletrencefalográficas. Em cada um dos casos estudados os resultados foram comparados com aquêles referidos na literatura.

\section{SUMMARY}

Clinical symptoms associated with metrazol activation in temporal lobe epilepsy.

Based on the metrazol activation of 25 cases of temporal lobe epilepsy, the author analyzes the varied clinical manifestations and electroencephalo- 
graphic sequence. In order to appreciate more readily the various patterns of temporal lobe seizures the major types of manifestations have been grouped in separate tabulations. The material suggests that there may be an ordered arrangement of certain clinical and electroencephalographic patterns concerned the temporal lobe.

\section{REFERENCIAS}

1. AJMONE-MARSAN, C.; RALSTON, B. L. - The Epileptic Seizure. A ClinicalElectrographic Analysis of Metrazol-induced attacks. Charles C. Thomas, Springfield (IIlinois), 1957. 2. AJMONE-MARSAN, C.; van BUREN, J. M. - Epileptiform activity in cortical and subcortical structure in the temporal lobe of man. In Temporal Lobe Epilepsy edit. por M. Baldwin e P. Bailey. Charles C. Thomas, Springfield (Illinois) 1958. 3. ARMBRUST-FIGUEIREDO, J. - Contribuição ao Estudo Clínico da Epilepsia Temporal. Tese de docência. Escola Paulista de Medicina, 1958. 4. BALDWIN, M.; FROST, L. L.; WOOD, C. D. - Investigation of the primate amygdala. Neurology $4: 586-593,1954$. 5. BRANDT, H. A.; JEUB, R. P. Definitive diagnosis in loss consciousness: Further experience with metrazol activation. Dis. Nerv. System 17:43-47, 1956. 6. BROSER, F.; HANN, J.; LEUBE, H. Die perorale Cardiozolmedikation als Epilepsietest: Untersuchungen an Eplleptiken unter EEG-Kontrolle. Nervenarzt 22:351-353, 1951. 7. CURE, C.; RASMUSSEN, T.; JASPER, H. - Activation of seizures and electroencephalographic disturbances in epileptic and in control subjects with metrazol. Arch. Neurol. \& Psychiat., 59:691717, 1948. 8. DELL, M. B. - L'epilepsie temporale. Presse Med., 61:505-508, 1953. 9. FUGLSANG-FREDERIKSEN, V. - Activation of EEG disturbances with Metrazol (Pentazol) in epileptics, normals and patients with syncopal attacks. Electroencephalog. \& Clin. Neurophysiol., 4:471-480, 1952. 10. GASTAUT, H.; NAQUET, R.; MEYER, A.; CAVANAGH, J. B.; BECK, E. - Clinical, Electroencephalographic and Anatomo-Pathological Study of "Psychomotor" Epilepsy Induced in the Cat. In Temporal Lobe Epilepsy, edit. por M. Baldwin e P. Bailey. Charles C. Thomas, Springfield (Illinois), 1958. 11. GASTAUT, H.; TOG, M.; NAQUET, R. - Clinical electrographical and anatomical study of epilepsy induced in dog by the ingestion of agenized proteins. In Temporal Lobe Epilepsy, edit. por M. Baldwin e P. Bailey. Charles C. Thomas, Springfield (Illinois), 1958. 12. GASTAUT, H.; VIGOROUX, M. - Electroclinical correlations in 500 cases of psychomotor seizures. In Temporal Lobe Epilepsy, edit. por M. Baldwin e P. Bailey. Charles C. Thomas, Springfield (Illinois), 1958. 13. GIBBS, E. L.; GIBBS, F. A.; FUSTER, B. - Psychomotor epilepsy. Arch. Neurol. \& Psychiat., 60:331-339, 1948. 14. GIBBS, F. A.; STAMPS, F. W. - Epilepsy Handbook. Charles C. Thomas, Springfield (Illinois), 1958. 15. JONES, E.; BAGCHI, B. K. - Orally administered metrazol combined with photic stimulation as an EEG activating procedure. Electroencephalog. \& Clin. Neurophysiol., 6:696-697, 1954. 16. KAUFMAN, F. C.; MARSHALL, C.; WALKER, A. E. - Metrazol activated electroencephalography. Proc. Am. Res. Nerv. \& Ment. Dis., 26:476, 1957. 17. MERLIS, J. K.; GROSSMAN, C.; HENRICKSEN, G. F. - Comparative effectiveness of sleep and Metrazol-activated electroencephalography. Electroencephalog. \& Clin. Neurophysiol., 3:71-78, 1951. 18. MERLIS, J. K.; HENRICKSEN, G. F.; GROSSMAN, C. - Metrazol activations of seizures discharges in epileptics with normal routine electroencephalograms. Electroencephalog. \& Clin. Neurophysiol., 2:17-22, 1950. 19. MOORE, F. J.; KELLAWAY, P.; KAGAWA, N. Metrazol activation as a diagnostic adjunct in electroencephalography; a re-evaluation. Neurology 4:325-338, 1954. 20. MULDER, D. M.; DALY, D. - Psychiatric symptoms associated with lesions of temporal Iobe. J.A.M.A., 150:173-176, 1952. 21. PAPEZ, J. W. - A proposed mechanism of emotion. Arch. Neurol. \& Psychiat., 38:725, 1937. 22. PENFIELD, W. - Epileptic automatism and the centroencephalic integrating system. Proc. Am. Res. Nerv. \& Ment. Dis., 30:513-528, 1952. 23. PENFIELD, W.; ERICKSON, T. C. - Epilepsy and Cerebral Localization. Charles C. 
'Thomas, Springfield (Illinois), 1941. 24. PENFIELD, W.; JASPER, H. - Epilepsy and the Functional Anatomy of the Human Brain. Little Brown, Boston, 1954.25. PEREZ-ACHARD, L.; GARCIA-ARUST, E. - Metrazol activation in epileptic and non-epileptic patients. Eletroencephalog. \& Clin. Neurophysiol., 6:536, 1954. 26. PEREZ-ACHARD, L.; GARCIA-ARUST, E.; GALEANO-MUÑOZ, C. - Metrazol activation in epilepsy. Electroencephalog. \& Clin. Neurophysiol., 8:153-154, 1956. 27. PUPO, P. P.; ZUKERMAN, E. - Some comparative results of activation methods in electroencephalography. Electroencephalog. \& Clin. Neurophysiol., 8:154, 1956. 28. RASMUSSEN, T.; JASPER, H. - Temporal lobe epilepsy: indication for operation and surgical technique. In Temporal Lobe Epilepsy, edit. por M. Baldwin e P. Bailey. Charles C. Thomas, Springfield (Illinois), 1958. 29. SMITH, B.; FAZEKAS, J.; FORSTER, F. M.; SMITH, G. E. - Activation of the electroencephalogram with oral pentylenetetrazol. Proc. III Internat. Congress EEG Clin. Neurophysiol., Cambridge (Mass.), 1953. 30. STEVENS, J. R. - The "march" of temporal lobe epilepsy. Arch. Neurol. \& Psychiat., 77:227-236, 1957. 31. STROBOS, R. J. - Mechanims in temporal lobe seizures. Arch. Neurol., 5:36-45, 1961. 32. TOWER, D. B. - The evidence for a neurochemical basis of seizures. In Temporal Lobe Epilepsy, edit. por M. Baldwin e P. Balley. Charles C. Thomas, Springfield (Illinois), 1958. 33. VICTOR, M.; ANGEVINE, V.; MANCALL, E. L.; FISHER, C. M. -- Memoryloss with lesions of hipocampal formation. Arch. Neurol., 5:244-263, 1961. 34. von MEDUNA, L. - Versuche über die biologische Beeinflussung des Ablaufes der Schizophrenia: Campher- und cardiazol Krampfe. Ztschr. Ges. Neurol. u. Psychiat., 152: 235, 1935. 35. WALKER, A. E. - Post-traumatic Epilepsy. Charles C. Thomas, Springfield (Illinois), 1949. 36. WEIL, A. A. - Ictal emotions occurring in temporal lobe dysfunction. Arch. Neurol., 1:87-97, 1959.

Clinica Neurológica, Faculdade de Medicina - Ribeirão Prêto, São Paulo - Brasil. 